\title{
Determinants of Obstetric Complications in Debre Behran Referral Hospital, Amhara Regional State, Ethiopia: A Matched Case-control Study
}

\author{
Abebe Balcha ${ }^{1,}$, , Robert Wondimu ${ }^{2}$, Zalalem Kaba ${ }^{3}$ \\ ${ }^{1}$ Kotu Primary Healthcare Unit, North Shewa Zone, Ethiopia \\ ${ }^{2}$ Sendafa Beke Town Health Office, Sendafa Beke Town, Ethiopia \\ ${ }^{3}$ East Wollega Zonal Health Office, Nekemte Town, Ethiopia \\ Email address: \\ abebeyor@gmail.com (A. Balcha), robertwondimu2021@gmail.com (R. Wondimu), kabazalalem@gmail.com (Z. Kaba) \\ ${ }^{*}$ Corresponding author
}

\section{To cite this article:}

Abebe Balcha, Robert Wondimu, Zalalem Kaba. Determinants of Obstetric Complications in Debre Behran Referral Hospital, Amhara Regional State, Ethiopia: A Matched Case-control Study. American Journal of Biomedical and Life Sciences.

Vol. 9, No. 3, 2021, pp. 151-165. doi: 10.11648/j.ajbls.20210903.13

Received: April 26, 2021; Accepted: June 4, 2021; Published: June 21, 2021

\begin{abstract}
Background: Obstetric complications are health problems that occur during pregnancy, delivery and postnatal period. In Ethiopia, obstetric complications are the major cause of maternal death in which results from represent a mix of risk factors such as illiteracy, lack of information, low use of health care, previous obstetric complications, previous chronic medical conditions, remote location, poverty and poor decision-making power of women. Objective: The aim of this study is to assess determinants of obstetric complications in Debre Berhan Referral hospital, Amhara Regional State, Ethiopia. Method: An institutional based matched case-control study was conducted on determinants of obstetric complications in Debre Berhan Referral Hospital from March 10 to April 30, 2019. Systematic sampling method was used to select 47 cases and 94 controls a total sample size of 141 and data was collected using pre-tested standardized questionnaire and checklist. Then data was entered and cleaned using Epi-info version 7.0, bivariate and multivariate analysis was done using conditional logistic regression on SPSS version 21. Descriptive statistics and odds ratio with $95 \% \mathrm{CI}$ was computed for predictor variables and Pvalue $<0.05$ was considered as significant. Results: Age 35 years and above [(COR=1.8, 95\% CI: (1.023-3.27)], age of first pregnancy less than 18 years [AOR=2.825, 95\% CI, (1.021-3.273)], lack information access [AOR=13.742, 95\% CI, (4.935$38.270)$ ], birth interval less or equal 1 year [AOR=2.581, 95\% CI, (1.679-3.896)]; past obstetric complications [AOR=3.450, 95\% CI, (2.001-4.155)], anemia [AOR=3.1, 95\% CI, (2.002-4.414)], focused ANC utilizations [AOR=0.30, 95\% CI, (0.014$0.877)$ ], and induced labor [AOR=4.9,95\% CI 1.008-7.321)] showed significant association with an obstetric complications. Conclusions and recommendations: ANC, age, birth interval, past obstetric complications, abortion, information access, educational status, planned pregnancy, anaemia, weight loss, and FP were determinants of obstetric complications in study area. Therefore, providing appropriate information on potential obstetric complications, accessed maternal health services and empower women is very important.
\end{abstract}

Keywords: Determinants, Obstetric Complications, Matched Case-control Study

\section{Introduction}

Obstetric complications are health problems that occur during pregnancy. They can involve the mother's health, the baby's health, or both. Some women have health problems that arise during pregnancy, and other women have health problems before they become pregnant that could lead to complications [1-3].

Obstetric complications are generally the symptoms and problems that are related to pregnancy and child birth. On the other hand complications during pregnancy and childbirth are the leading cause of disability and death among women between the ages of 15-49 years. Many women face some minor health problems while some women who unfortunately get faced with 
more serious complications during pregnancy [4].

Maternal mortality is unacceptably high. About 830 women die from pregnancy- or childbirth-related complications around the world every day. It was estimated that in 2015, roughly 303,000 women died during and following pregnancy and childbirth. Almost all of these deaths occurred in low-resource settings, and most could have been prevented [5].

In India the nationwide survey covering 601 districts from 34 states and union territories in 2015 about complications during pregnancy described that 44 present of the women had reported complications like paleness, giddiness or weakness during pregnancy. Excessive fatigue, swelling of hands, feet and face, vomiting were also found as the most reported complications among Indian women during pregnancy. Less than one fifth of women were reporting of visual disturbance mainly caused by chronic vitamin A deficiency. It has found from the analysis that a few percentage of the studied women were reported of having high blood pressure, vaginal discharge, malaria, abnormal position of foetus, and bleeding during her pregnancy period [6].

Study in Uganda also shows the socio-demographic characteristics and medical history of the pregnant women that stratified by severity of complications; in relation to the childbirth event, the most likely time for a mother to develop severe obstetric complications were if they occurred in the intra partum period and continued in the postpartum period [7].

About 20,000 women also die each year in Ethiopia from complications during pregnancy, childbirth and in the postpartum period. The national maternal death surveillance report (MDSR) of Ethiopia in 2016 stated that among 631 maternal deaths a large majority of deaths $(82 \%)$ were attributed to direct causes, while indirect causes were reported for $13 \%$. In $32(5 \%)$ cases the cause of death was not specified (reported as "unknown") except for the fact that it was unequivocally obstetric. From such causes haemorrhage was the leading direct cause of death, accounting for $49 \%$ of direct causes and $42 \%$ of all causes, followed by pre-eclampsia or eclampsia (19\%), obstructed labour/ruptured uterus (9\%) and infections $(7 \%)[8,9]$.

Maternal health outcomes are known to be influenced by a range of multi-sector determinant factors. Government policies and actions regarding maternal health, general health services, and systems, access to important sectors such as education and transport, socio-cultural beliefs, norms, and practices, and community and household behaviours regarding pregnancy are just a few of them. All these determinant factors have their impending role either in severely restricting or in positively enhancing good maternal health. The health status which is consisted of nutritional status, anaemia, disease history and previous pregnancy complications also has great impact on current pregnancy $[10$, $11]$.

Generally the international evidence suggests that other determinant factors such as ANC follow up, literacy, access to income and health service and early referral which can directly reduce maternal obstetric complications are improving the quality of obstetric care. Accurate and reliable quality of obstetric cares is important on the quality of services provided at health facilities in Ethiopia, especially for complicated deliveries involving postpartum haemorrhage and pre-eclampsia or eclampsia [12].

Obstetric complication and their consequences have high effects on a pregnant mother or her foetus during pregnancy, delivery, and up to 42 days postpartum period. Globally, about eight million women having pregnancy-related complications (obstetric complications) and more than half a million die from those complications in every year [13].

Many research finding shows that, more than half of the studied women were suffering from any type of complications during pregnancy. It also shows a relationship of obstetric complications on adverse birth outcome. It has found from the study that women with any obstetric complications had a more chances of delivering still birth compared to women without any complication [14].

According to the communicable diseases control (CDC), severe maternal morbidity (obstetric complication) has risen faster than maternal mortality. Based on the rate per 10,000 deliveries, serious complications more than doubled from 1993 to 2014, driven largely by a five-fold rise in blood transfusions. That also includes a nearly 60 per cent rise in emergency hysterectomies (removal of the uterus) and sometimes other reproductive organs, often to stem massive bleeding or infection. In 2014 alone, more than 4,000 women had emergency hysterectomies, rendering them permanently unable to carry a child. The rates of new mothers requiring breathing tubes, and of treatment for sepsis both increased by 75 per cent. And the rate of women needing to be resuscitated from heart failure rose by 175 percent [15].

The most severe obstetric complications of pregnancy, affect more than 50,000 women in the United States every year. Based on recent trends, this burden has been steadily increasing. Rises in obstetric complications are likely driven by a combination of factors, including increases in maternal age, pre-pregnancy obesity, pre-existing chronic medical conditions, and caesarean delivery. The consequences of the increasing obstetric complications prevalence are wideranging and include higher health service use, higher direct medical costs, extended hospitalization stays, and long-term rehabilitation [1].

High rates of maternal mortality due to obstetric complications still exist in places, particularly in impoverished communities with over $85 \%$ living in Africa and Southern Asia. The effect of a mother's death results in vulnerable families and their infants, if they survive childbirth, are more likely to die before reaching their second birthday $[16,17]$.

In Ethiopia the obstetric complications are strongly increased and become the major cause of maternal death. About fifteen percent of pregnant women in Ethiopia are estimated to develop obstetric complications which are potentially life-threatening. From maternal death direct complication accounts for $85 \%$ of the deaths as well as which 
leads to many acute and chronic illnesses including disabilities. Even if maternal mortality levels due to obstetric complications declined significantly in Ethiopia during the last two decades, the achievement still fell short of the country target to reach and MMR 267/100,000 live births by 2015. Hence, maternal health continues to be a public health priority a new national target has been set to reduce maternal mortality to $199 / 100,000$ live births by the end of 2020 . Improving the quality and equity of maternity services is thus the primary focus in the Health Sector Transformation Plan strategy for the period 2016 to 2020 [10].

According to the national MDSR 2016 of Ethiopia, the majority of reported deaths around sixty eight present (68\%) occurred in health facilities with the following distribution: $63 \%$ in hospitals and $5 \%$ in health centres in which the majority contributing causes were obstetric complications [10].

On the other hand, I haven't get any official studies concerned determinants of obstetric complication in Debre Berhan Referral hospital and other similar setting in North Shewa zone, Amhara regional state, Ethiopia. So in the light of this background; therefore the present study examined the determinant factors that brings obstetric complications and tries to fill the gap on scarcity of information, evidence and base line. The data was also generated to assist the local authority and Debre Berhan Referral hospital administrators by being the source of information and evidence to take measures and making plan.

\section{Methods and Materials}

\subsection{Study Area and Period}

This research was conducted in Debre Berhan Referral hospital which is found in Debre Berhan town (the capital city of North Shewa zone, Ethiopia). The town is found in Amhara regional state, North Shewa Zone and it is 130 kilometres (Kms) far from Addis Ababa (which is the capital city of Ethiopia) to the north east. The town has cool weather condition throughout the year with the average temperature of 14 degree centigrade and $86 \%$ humidity, and found at 9.68 degree latitude and 39.53 degree longitude. Debre Berhan Referral hospital has 4 major wards (Gynaecology and Obstetrics ward, Medical ward, surgical ward and Paediatrics ward); Concerning maternal and neonatal service it has three outpatient departments (OPD) such as maternal and child health department, family planning service department, and post natal service; and it has also Pharmacy department, Emergency department, and $\mathrm{MCH}$ department and Laboratory department. From such wards and departments, the Gynaecology and Obstetrics wards and the MCH departments directly serve the maternal health. The hospital providing both Comprehensive Emergency Obstetric care (CEmOC) and Basic Emergency Obstetric Care (BEmOC) functions. The hospital serves for more than 2.5 million estimated populations and has in average more than 1,000,000 patient flows per year. As the quarter reports of the hospital indicates that in average more than 100 mothers with obstetric complications and more than 1,000 mothers without obstetric complication have gotten service in gynaecology and obstetrics ward as well as in $\mathrm{MCH}$ outpatient department in every three months [18]. The study was conducted from March 10 to April 30, 2019.

\subsection{Study Design}

1. The study was conducted using an institutional based matched case-control study design.

2. A cases in this study were any pregnant and up to 42 postpartum days woman aged 15-49 years who had suffered from at least one obstetric complication (Haemorrhage, PROM or chorioamnionities, Hyper Emesis gravid arum, Pregnancy induced hyper tension, Obstructed labour or Obstetric fistula, Mal-presentation, Uterine rupture /Imminent uterine rupture, Abortion, Poly-hydaminus or oligo-hydraminus and Intra uterine growth restriction (IUGR)) or gave birth by Augmentation, Induction or caesarean section due to obstetric complications while in pregnancy; labour, during delivery or within 42 days after delivery that necessitated treatment, referral or hospitalization in Debre Berhan Referral Hospital during the study period.

3. Controls in this study were any pregnant and up to 42 postpartum days women aged 15-49 years who had come to DRH in obstetric or gynaecology ward for services during the study period but have no obstetric complications.

4. Matching:-cases and controls were matched by parity, and place of current residence (rural or urban) on the ratio of one case for two controls.

\subsection{Populations}

\subsubsection{Source Population}

The source population were all pregnant and up to 42 postpartum days' women who attended Obstetrics and Gynaecology Department in Debre Berhan referral hospital from March 10 to April 30, 2019.

\subsubsection{Study Population}

1. Study population for cases were those pregnant and up to 42 postpartum days' women who attended Obstetrics and Gynaecology ward and have recorded diagnose of obstetric complications in Debre Berhan referral hospital from.

2. Study population for controls were those pregnant and up to 42 postpartum days' women without obstetric complications who attended Obstetrics and Gynaecology ward and have records for service in Debre Berhan referral hospital.

\subsection{Eligibility Criteria}

\subsubsection{Exclusion Criteria}

\section{(i) Exclusion Criteria for Cases}

Pregnant women with obstetric complications but with incomplete records were excluded. 


\section{(ii) Exclusion Criteria for Controls}

Pregnant women without obstetric complications who will come for gynaecologic or obstetric services but with incomplete records were excluded.

\subsection{Sample Size Determination and Sampling Procedure}

\subsubsection{Sample Size Determination}

The Sample size is estimated using a double population proportion formula assuming 3.5\% proportion of control and $31.4 \%$ proportions of case based on the study conducted in Jimma University Specialized Hospital that showed that the determinants of obstetric complications among mothers by assuming pregnant mothers who are anaemic or had haemoglobin level of less than $11 \mathrm{gram} / \mathrm{dl}$ are more than seven times to have obstetric complication than non-anaemic pregnant mothers, (31); 95\% confidence level and 80\% power of the test with a case to control the ratio of $1: 2$. From this statement

$\mathrm{P} 1$ (case) $=0.314$ where " $\mathrm{p}$ " is proportion

P2 (control) $=0.035, \mathrm{CI}=95 \%$, Power of the test $=80 \%$, $r=1: 2$ (where " $r$ " is ratio of case to control. Then

$$
\mathrm{n}=\left(\frac{\mathrm{r}+1}{\mathrm{r}}\right) \frac{\left(\mathrm{p}^{-}\right)\left[1-\mathrm{p}^{-}\right][\mathrm{z} \beta+\mathrm{z} \alpha / 2]^{2}}{(\mathrm{p} 1-\mathrm{p} 2)^{2}}
$$

Where:- $\mathrm{n}=$ Calculated sample size, $\mathrm{r}=$ ratio of control to case $=2: 1, \mathrm{P}^{-}=$average proportion of case and control in which $\mathrm{p}^{-}=\frac{\mathrm{p} 1+\mathrm{p} 2}{2}$ $\mathrm{p} 1=$ proportion of case when $\mathrm{p} 1=\frac{\mathrm{OR}(\mathrm{p} 2)}{\mathrm{p} 2(\mathrm{OR}-1)+1}$

$\mathrm{p} 2=$ proportion of control $=0.035$ from the literature

$\mathrm{Z} \beta$ for $80 \%=0.84, \mathrm{Z} \alpha / 2=1.96 ; \mathrm{N}=42+10 \%$ non- response rate 47 cases were considered. Since ratio of case to control is $1: 2$ the sample size of control is 94 . Accordingly, the final sample size was 47 cases and 94 controls (a total sample size of 141) were involved in the study.

\subsubsection{Sampling Technique and Procedure}

After reviewing all records, the first sample of a case and two controls were selected by using simple random sampling (SRS) method then a systematic sampling technique was used to select the whole study units. All records of pregnant women at Debre Berhan Referral Hospital during each days of study period were reviewed using delivery room, operation room and postnatal ward log books. Then women who had at least one obstetric complication and/or gave birth by Augmentation, Induction or caesarean section due to obstetric complications were selected and recorded to the study units of the cases. In the same way, women who attend but had no any obstetric complications also were selected and recorded for the study units as the control. After reviewing, systematic sampling method was used to collect the whole data by selecting the every other case and controls based on their attending orders. Matching was applied with the case on the basis of parity and residence in the ratio of for one case; two controls. The procedure was continues until the total sample had been met.

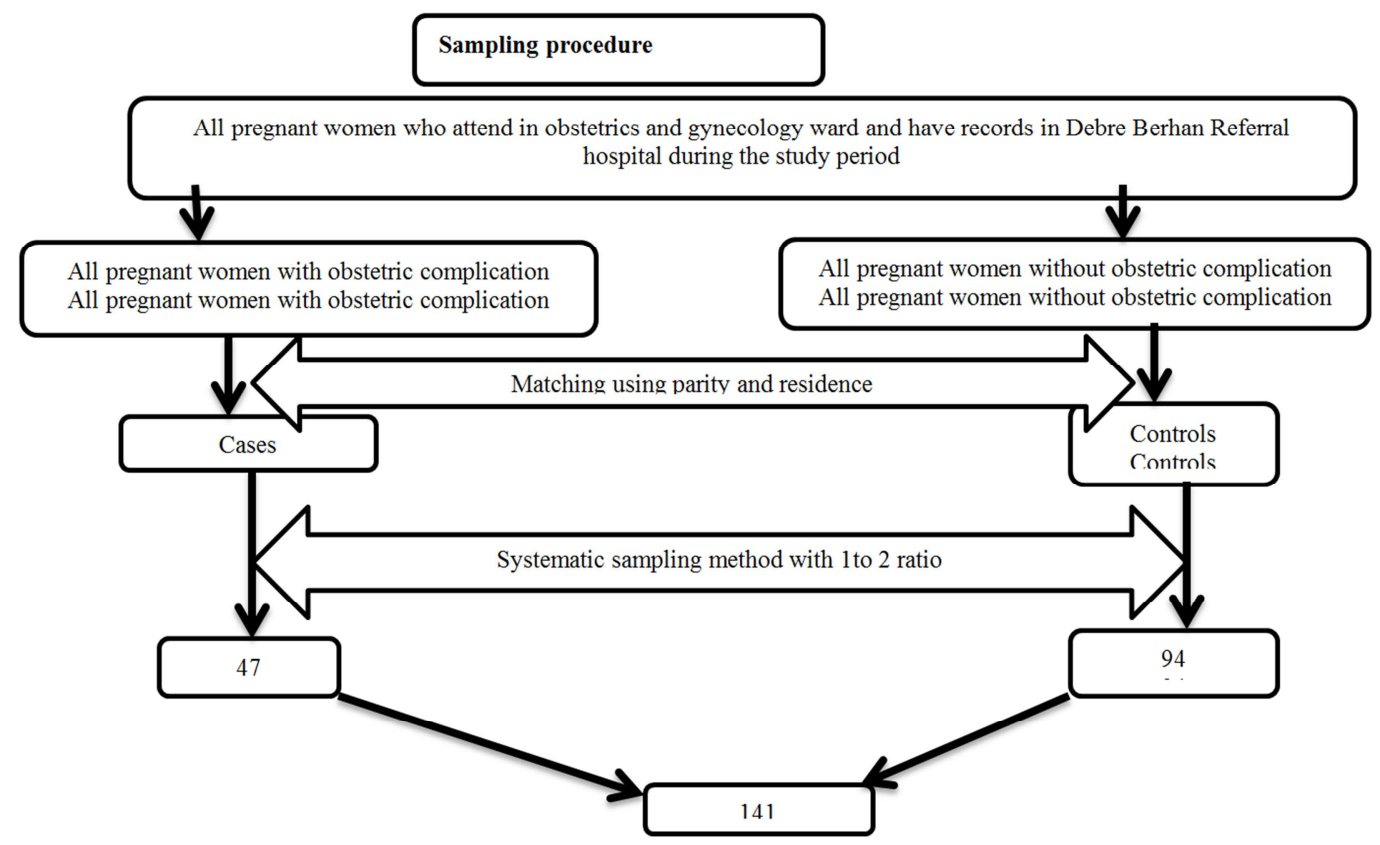

Figure 1. Diagrammatic representations of sampling procedure for the study of obstetric complications, Debre Berhan, Ethiopia, April 2019.

\subsection{Study Variables}

\subsubsection{Dependent Variables}

Obstetric complications

\subsubsection{Independent Variables}

Distant determinant (socio demographic) variables:
1. Women's related variables:-Age, Education, Occupations, and Income

2. Husbands related variables:-Education, and Occupation

3. Infra-structure related variables:-Health institution (access to ANC, PNC and FP services)

Intermediate variables 
1. Health related variables:- Anaemia, Hepatitis, Intestinal parasites, Hypertension, Diabetic mellitus, Weight loss, Cardiac problems, and Renal problems

2. Reproductive health related variables:-Marital status, Age during time of pregnancy, Birth interval, Abortion, Still birth, and History of previous complications

3. Access to information:- Mass media, and Health education

4. Health service utilization related variables:-Focused ANC, FP, Skill delivery, and PNC

\subsection{Operational Definitions}

1. Ante partum haemorrhage: bleeding from the vaginal tract of the pregnant mother after the foetus has reached the age of viability which is after 28 completed gestational age and before the foetus is delivered.

2. Augmentation: enhancing uterine contractions that are inadequate to facilitate progress of labour.

3. Chronic condition: condition/diseases which is presented before pregnancy and persists after 6 weeks of postpartum.

4. Grand multipara: A mothers whose age equal/greater 35 years old and born 3 or more children.

5. Distal determinants: are socio-demographic variables of mothers and partners that are strongly influence woman to develop obstetric complications in the society.

6. Induction: initiation (stimulation) of uterine contractions artificially for the purpose of delivering the foetus vaginally after the foetus has reached viability (after the 28 weeks of gestation)

7. Intermediate determinants: are factors which are reproductive health, chronic disease and health service utilization related variables of pregnant woman which directly affect to develop obstetric complications.

8. Focused ANC service utilizations: mothers who receive ANC service in health institution at list 4 times up to their labour, and receive the first service before 16 weeks, second service 24-32 weeks, third service at 32 weeks and last 36 weeks of gestation.

9. Mal-presentation: abnormalities of foetal positions, presentations, attitude or lie.

10. Maternal Death: the death of the woman while pregnant or within 42 days of terminations of pregnancy irrespective of the duration of pregnancy from any cause related to or aggravated by pregnancy or its management but not from accidental or incidental cause.

11. Obstetric complications: Presence of one of the following: Ante partum haemorrhage (APH), PROM or chorioamnionities, preeclampsia or eclampsia, obstructed labour, mal-presentation, suspected uterine rupture, previous caesarean delivery or gynaecology operations.

12. Obstructed labour: is failure of descent of the foetus in the birth canal for the mechanical reasons arising from either the passage or passenger in spite of adequate uterine contraction.
13.Parity: foetus delivered after 28 weeks of gestation.

14. Postpartum haemorrhage: Is defined as estimated blood loss of $>=1000 \mathrm{ml}$, fall in Hct $>10 \%$, post-operative Hct $<25 \%$ or bleeding that is greater than expected and results in signs and/or symptoms of hypervolemia after the fetus delivered to 42 postpartum days.

15. Preeclampsia: occurrence of hypertension, proteinuria, and/or edema after 20 completed weeks of gestation and resolves within 6 weeks of postpartum.

16. Eclampsia: tonic colonic convulsion or coma occurring during pregnancy, labor or within 7 days of postpartum unrelated to other cerebral conditions like epilepsy in a woman with neglected or fulminate preeclampsia.

17. Premature rupture of membrane: rupture of membrane at least one hour of before the onset of labor after 28 weeks of gestations.

18. Primigravida: A mother who experience pregnancy for the first time

19. Suspected uterine rupture: the imminent uterine rupture that worsening abdominal pain especially supra pubic persisting between contraction and strange feeling of the fetus moving upward.

\subsection{Data Collection Methods and Tools}

Standardized questionnaire and check lists adapted from Obstetric complications, emergency caesarean delivery outcome tracing tools in order to identify determinants of obstetric complications was used for data collection [16, 1920]. Data was collected using pre-tested standardized check lists and questionnaire. Accordingly, 10 questions were developed to address the socio-demographic characteristics. For the reproductive health characteristics, current obstetric complications and determinant factors related, 10, 12 and 25 questions were developed respectively. A total of 58 questions (using interviews and observational check lists) were used. The data collection methods were face-to-face interviews on socio demographic characteristics, reproductive health related and determinant factors parts; and observational check lists of registers or log books current obstetric complications part during antenatal period, delivery and post-natal period. For each case identified and recruited, selection and recruitment of an appropriate two control from the pregnant or 42 postpartum days women who didn't develop obstetric complications within the same residence (rural or urban) and parity was conducted. Thus, procedure was continued until the required sample size was achieved. The data collectors were health professionals. One day training was provided to for two clinical nurse data collectors and one midwifes supervisors to equip them with basic knowledge and skills of data collection and related procedures. Before initiation of data collection, the purpose of the study was also explained to the obstetric staffs, medical directors and study participants.

\subsection{Data Quality Assurance}

To assure quality of data, a standardized questionnaire and 
checklist adapted from Obstetric Complications, emergency caesarean delivery outcome tracing tools was used for this study. The check lists and questionnaire was first translated in to Amharic before data collection then it retranslated back to English after data collection and before analysis to check for consistency by experts. However, to meet the objectives of the study, the Check lists/ questionnaire was carefully modified slightly in line with the context. It was designed in the way that to collect information about the relevant variables. Then one week prior to the actual data collection, questioner and checklists was pre tested on $5 \%$ of the study participants that is 3 cases and 6 controls totally 9 participants in Deneba District hospitals for its clarity, understand ability and completeness. Check lists/ questionnaire was checked thoroughly for its completeness, objective and variable based before it distributed to respondents. During the actual data collection process, there was cross checking randomly every day for checklists consistency and completeness before entry to computer software for analysis.

\subsection{Data Management and Analysis}

Data was entered and cleaned by using EPI info version 7.0 and the cleaned data was exported to SPSS version 21 for further analysis purpose. Descriptive statistical analyses including frequency distribution, measures of central tendency, and dispersion were computed. To identify factors associated with obstetric complications bivariate analysis in conditional logistic regression was used to see the association between one explanatory variable and outcome variable at $95 \%$ confidence intervals along with their $p$-value $<0.25$. Multivariable analysis in conditional logistic regression was performed to predict factors which affect the dependent variable. Those variables with $\mathrm{p}$ value $\leq 0.05$ were considered statistically significant in multivariable analysis. Overall findings were presented using texts, graphs and tables.

\subsection{Ethical Considerations}

Prior to starting the study, the final proposal was submitted to Department of public health, College of Health Science, Debre Berhan University for ethical clearance and approval. Then letter of permission was obtained from the Ethical review committee of Department of public health, College of Health Science, Debre Berhan University. After explaining about the purpose of the study, and the possible benefit of the study; oral permission was obtained from the hospital director before proceeding. Respect and full confidentiality of the study participant's response was granted since only the investigator was having access to the data. Then information was collected after securing oral consent from study participant. Individual Verbal consent was secured as well.

\section{Results}

\subsection{Socio Demographic Characteristics}

A total of 141 mothers with $100 \%$ response rate were interviewed. Overall, 47 cases were matched with 94 controls on parity and residence (rural and urban). The mean age of cases and controls were $31.15( \pm 8.25)$ and $29.35( \pm 6.5)$ years respectively. The majority of respondents both in case and control $(74.5 \%$ and $87.2 \%),(63.8 \%$ and $63.8 \%)$, and $(87.2 \%$ and 92.6\%) were Amara, orthodox and married respectively. More than seventy three percent of controls and $26.2 \%$ of cases of mothers was government employer [Table 1].

Table 1. Socio-demographic characteristics of the study participants in Debre Berhan Referral Hospital, Debre Berhan Town, Ethiopia, June 2019.

\begin{tabular}{|c|c|c|c|c|}
\hline Variables & Categories & Case $n=47(\%)$ & Controls n=94 (\%) & Totals n=141 (\%) \\
\hline \multirow{3}{*}{ Age (in years) } & $15-24$ & $10(21.3)$ & $26(27.7)$ & $34(24.8)$ \\
\hline & $25-34$ & $25(53.2)$ & $58(61.7)$ & $84(59.6)$ \\
\hline & $\geq 35$ & $12(25.5)$ & $10(10.6)$ & $22(15.6)$ \\
\hline \multirow{2}{*}{ Ethnicity } & Amara & $35(74.5)$ & $82(87.2)$ & $117(83)$ \\
\hline & Others & $12(24.5)$ & $12(12.8)$ & $24(17.0)$ \\
\hline \multirow{4}{*}{ Religion } & Orthodox & $30(63.8)$ & $60(63.8)$ & $90(63.8)$ \\
\hline & Muslim & $9(19.1)$ & $11(11.7)$ & $20(14.2)$ \\
\hline & Protestants & $8(17.1)$ & $16(17.1)$ & $24(17)$ \\
\hline & Catholic & - & $7(7.4)$ & $7(5)$ \\
\hline \multirow{3}{*}{ Marital status } & Single & $2(4.3)$ & $4(4.3)$ & $6(4.3)$ \\
\hline & Married & $41(87.2)$ & $87(92.6)$ & $128(90.8)$ \\
\hline & Divorced & $4(8.5)$ & $3(3.1)$ & $7(5)$ \\
\hline \multirow{4}{*}{$\begin{array}{l}\text { Husbands educational status } \\
\text { (Case } n=43 \text {, Control } n=87 \text { ) }\end{array}$} & No formal education & $18(42.9)$ & $7(8.0)$ & $25(19.5)$ \\
\hline & Primary (1-8 grade) & $6(14.3)$ & $15(17.0)$ & $21(16.2)$ \\
\hline & Secondary (9-12 grade) & $10(23.8)$ & $37(42)$ & $47(36.2)$ \\
\hline & College diploma and above & $9(19.0)$ & $29(33)$ & $37(28.5)$ \\
\hline \multirow{4}{*}{$\begin{array}{l}\text { Husbands Occupation } \\
\text { (Case } n=43 \text {, Control } n=87 \text { ) }\end{array}$} & Student & $23(53.5)$ & $38(43.7)$ & $61(46.9)$ \\
\hline & Farmer & $8(18.6)$ & $28(32.2)$ & $36(27.7)$ \\
\hline & Government employ & $11(25.6)$ & $20(23.0)$ & $31(23.8)$ \\
\hline & Merchant & $1(2.3)$ & $1(1.1)$ & $2(1.5)$ \\
\hline \multirow{4}{*}{ Monthly income } & $<600$ ETB & $16(34.8)$ & $18(18.9)$ & $34(24.1)$ \\
\hline & 600-1000ЕТВ & $3(6.5)$ & $12(12.6)$ & $15(10.6)$ \\
\hline & 1000-2000ЕТВ & $7(15.2)$ & $14(14.7)$ & $21(14.9)$ \\
\hline & $>2000 \mathrm{ETB}$ & $21(43.5)$ & $50(53.7)$ & $71(50.4)$ \\
\hline
\end{tabular}




\subsection{Current Obstetric Complications}

Hemorrhage is the major obstetric complication in this study $(43.5 \%)$ which followed by obstructed labor (37\%) and hypertensive disorder of pregnancy $(23 \%)$ respectively. Out of 20 hemorrhage case $(75 \%)$ were postpartum hemorrhage whereas the rest was antepartum hemorrhage. In the same way from the hypertensive disordered of pregnancy cases about $55 \%$ were preeclampsia which followed bay ecalmpsia and super imposed chronic hypertension [Figure 2].

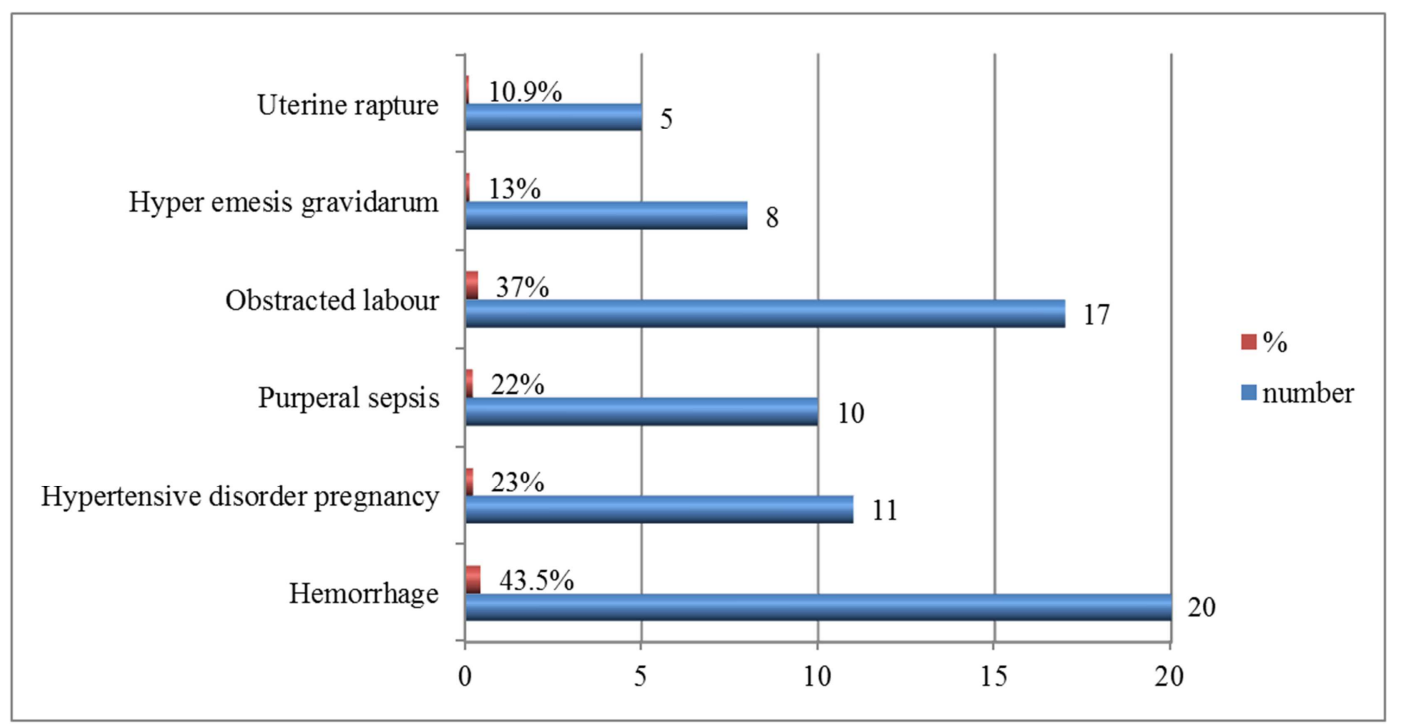

Figure 2. Current obstetric complications among women in Debre Berhan Referral Hospital, Debre Berhan Town, Ethiopia, June 2019

\subsection{Reproductive Characteristics of Women}

The mean gravidity, parity and birth interval of cases were $2.67( \pm 2.18), 2( \pm 1.63)$, and $3.65( \pm 2.9)$, and mean gravidity, parity and birth interval of controls were $2.35( \pm 1.39), 1.94$ $( \pm 1.5)$, and $2.7( \pm 0.9)$ respectively. The mean gestational age during the occurrence of obstetric complications of cases was $35.2( \pm 8.6)$ weeks which ranges from $32-43$ weeks. This study shows that more than half of the participants had 2-4 gravidities $72(51.1 \%)$; and most of the cases $30(63.8 \%)$ and about $34(35.2 \%)$ controls of study participants had $\geq 4$ years birth intervals. On the other hand about $21.7 \%$ cases and only $4.2 \%$ controls had less and equal 1 year birth interval in their last pregnancy [Table 2].

Table 2. Reproductive characteristics of the study participants in Debre Berhan Referral Hospital, Debre Berhan Town, Ethiopia, June 2019.

\begin{tabular}{|c|c|c|c|c|}
\hline Variables & Categories & Case $n=47(\%)$ & Controls n=94 (\%) & Totals $n=141(\%)$ \\
\hline \multirow{3}{*}{ Gravidity (in numbers) } & 1 & $18(38.3)$ & $30(31.9)$ & $48(34)$ \\
\hline & $2-4$ & $21(44.7)$ & $51(54.3)$ & $72(51.1)$ \\
\hline & 5 and above & $8(17.0)$ & $13(13.8)$ & $21(44.9)$ \\
\hline \multirow{3}{*}{ Birth interval } & $\leq 1$ year & $10(21.7)$ & $4(4.2)$ & $14(9.9)$ \\
\hline & 2-4 year & $7(14.8)$ & $56(60)$ & $63(44.7)$ \\
\hline & $\geq 4$ years & $30(63.8)$ & $34(35.2)$ & $64(45.4)$ \\
\hline \multirow{2}{*}{ Age at first marriage (Cases $n=43$, Controls $n=88$ ) } & $<18$ years & $11(24.6)$ & $1(1.1)$ & $12(9.1)$ \\
\hline & $\geq 18$ years & $32(74.4)$ & $87(98.9)$ & $119(90.9)$ \\
\hline \multirow{2}{*}{ Age at first pregnancy } & $<18$ years & $9(19.1)$ & $1(1.1)$ & $10(7.1)$ \\
\hline & $\geq 18$ years & $38(80.9)$ & $93(98.9)$ & $131(92.9)$ \\
\hline \multirow{2}{*}{ History of previous obstetrics complication } & Yes & $13(26.7)$ & - & $13(9.21)$ \\
\hline & No & $34(73.9)$ & $94(100)$ & $141(90.8)$ \\
\hline \multirow{2}{*}{ History of abortion } & Yes & $7(13.0)$ & $5(16.3)$ & $12(8.5)$ \\
\hline & No & $40(87)$ & $29(93.7)$ & $129(91.5)$ \\
\hline History of still birth & Yes & $4(6.5)$ & $3(1.1)$ & $7(2.9)$ \\
\hline \multirow{2}{*}{ Induced labor } & Yes & $12(26.5)$ & $2(2.1)$ & $14(9.9)$ \\
\hline & No & $35(73.9)$ & $92(97.9)$ & $127(90.1)$ \\
\hline \multirow{2}{*}{ Multiple pregnancy } & Yes & $5(8.7)$ & $5(6.3)$ & $10(10.1)$ \\
\hline & No & $42(91.3)$ & $89(93.7)$ & $131(92.9)$ \\
\hline
\end{tabular}

Regarding gave birth, forty respondents of cases had experience of gave birth in their life whereas the rest experiences pregnancy for the first time. Among them more than half $21(53 \%)$ respondents delivered their last child in 
health facility [Figure 3].

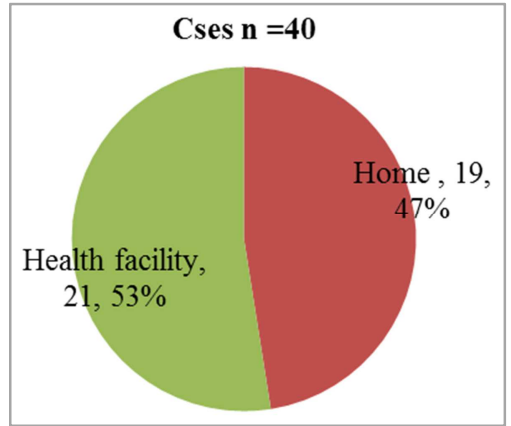

Figure 3. Place of last birth of Case respondents in Debre Berhan Referral Hospital, Debre Berhan Town, Ethiopia, June 2019.

Regarding controls, eighty four respondents gave birth experience previously; among them most of $74(88 \%)$ respondents delivered their last child in health facility while the rest delivered at home [Figure 4].

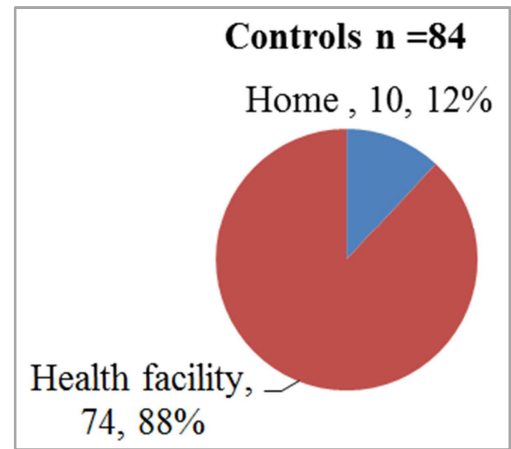

Figure 4. Place of last birth of Control respondents in Debre Berhan Referral Hospital, Debre Berhan Town, Ethiopia, June 2019.

Among 40 cases and 84 controls who gave birth recently; majority controls $80(95.2 \%)$ gave birth by spontaneous vaginal delivery (SVD); whereas most cases $22(55 \%)$ gave birth by assisting instruments. Regarding cesarean delivery, about $30 \%$ cases delivered by emergency cesarean surgery; whereas no one controls had CS delivery [Figure 5].



Figure 5. Mode of recent delivery of respondents in Debre Berhan Referral Hospital, Debre Berhan Town, Ethiopia, June 2019.

\subsection{Health Conditions}

Of those cases about $19.6 \%$ and $17.4 \%$ had history of anemia and weight loss respectively whereas none of controls had history of anemia but $3.2 \%$ weight loss in recent pregnancy. According to this study women with obstetric complications (cases) had more weight loss than women who hadn't obstetric complications (controls) i.e. 17.4\% and 3.2\% respectively. Hypertension is the majority chronic condition $(41.7 \%)$ which followed by Diabetic Mellitus $(25 \%)$ and Hepatic problem (25\%) in this study [Table 3]. 
Table 3. Health related factors of the study participants in Debre Berhan Referral Hospital, Debre Berhan Town, Ethiopia, June 2019.

\begin{tabular}{lllll}
\hline Variables & Categories & Cases $(\mathbf{n}=\mathbf{4 7})$ & Controls $(\mathbf{n}=\mathbf{9 4})$ & Total $(\mathbf{n}=\mathbf{1 4 1})$ \\
\hline \multirow{2}{*}{ Anemia } & Yes & $10(19.6)$ & - & $10(7.1)$ \\
& No & $37(80.4)$ & $94(100)$ & $131(92.9)$ \\
Weight loss & Yes & $9(17.4)$ & $2(3.2)$ & $11(7.8)$ \\
& No & $38(82.6)$ & $92(96.8)$ & $130(92.2)$ \\
Infection & Yes & $10(19.6)$ & $23(25.3)$ & $23(23.4)$ \\
& No & $37(80.4)$ & $71(74.7)$ & $108(76.6)$ \\
Chronic condition & Yes & $12(23.9)$ & $10(11.6)$ & $22(15.6)$ \\
& No & $35(76.1)$ & - & $119(84.4)$ \\
Types of chronic conditions & DM & $3(25.0)$ & - & $4(18.3)$ \\
(Case n=12, Controls n=10) & Hypertension & $5(41.7)$ & $9(90.0)$ & - \\
& Cardiac problems & - & $1(10.0)$ & $9(40.9)$ \\
& Renal problems & $1(8.3)$ & - & $2(9.1)$ \\
\hline
\end{tabular}

\subsection{Health Service Access, Utilization, and Access to Information}

Majority of the controls, $73(77.9 \%)$ and 25 (52.2\%) cases had planned pregnancy. The proportion of women who received focused ANC service was higher among controls 93
(98.9\%) than cases 23 (49\%). Among 94 controls only $1.1 \%$ whereas out of 47 cases about $32.6 \%$ hadn't access to ANC service, and most of cases couldn't get information access regarding obstetric complications $34(72.3 \%)$ in contrast most of control could get information access regarding determinants of obstetric complications [Table 4].

Table 4. Health service, utilizations and information related factors of the study participants in Debre Berhan Referral Hospital, Debre Berhan Town, Ethiopia, June 2019.

\begin{tabular}{|c|c|c|c|c|}
\hline Variables & Categories & Cases $(n=47)$ & Controls $(n=94)$ & Total $(n=141)$ \\
\hline \multirow{2}{*}{ Availability of FP service } & Yes & $36(76.1)$ & $94(100)$ & $130(92.2)$ \\
\hline & No & $11(23.9)$ & - & $11(7.8)$ \\
\hline FP utilization & Yes & $29(60.9)$ & $88(93.7)$ & $117(83)$ \\
\hline \multirow{2}{*}{ Availability of ANC service } & Yes & $32(67.4)$ & $93(98.9)$ & $125(88.7)$ \\
\hline & No & $15(32.6)$ & $1(1.1)$ & $16(11.3)$ \\
\hline ANC utilization & On WHO recommendation & $23(49)$ & $93(98.9)$ & $116(82.3)$ \\
\hline \multirow{2}{*}{ Availability of PNC } & Yes & $34(71.7)$ & $93(98.9)$ & $127(90.1)$ \\
\hline & No & $13(28.3)$ & $1(1.1)$ & $14(9.9)$ \\
\hline \multirow{2}{*}{ PNC utilization } & On WHO recommendation & $10(21.7)$ & $51(53.7)$ & $61(43.3)$ \\
\hline & Not on WHO recommendation & $37(78.3)$ & $43(46.3)$ & $80(56.7)$ \\
\hline \multirow{2}{*}{ Planned pregnancy } & Yes & $25(52.2)$ & $73(77.9)$ & $98(69.5)$ \\
\hline & No & $22(47.8)$ & $21(22.1)$ & $43(30.5)$ \\
\hline \multirow{2}{*}{ Information access } & Yes & $13(27.7)$ & $82(87.2)$ & $95(67.4)$ \\
\hline & No & $34(72.3)$ & $12(12.8)$ & $46(32.6)$ \\
\hline
\end{tabular}

\subsection{Determinant Factors of Obstetric Complications}

\subsubsection{Distant Determinants}

\section{(i) Bivariate Analysis of Distant Determinant Factors Associated with Obstetric Complication}

In bivariate analysis was run in the conductional logistic regression show that the odds of Pregnant women with age 35 and above were 1.8 times higher to develop obstetric complications $[\mathrm{COR}=1.83,95 \% \mathrm{CI},(1.023-3.27)]$ compared to their counter parts; mothers who had experience of first pregnancy less than 18 years in current pregnancy 8.4 times more likely develop obstetric complication than counterparts; $[\mathrm{COR}=8.364,95 \% \mathrm{CI}$, (8.02-9.07)]; who had no formal education 3.8 more likely develop obstetric complications than mothers who had formal education, $[\mathrm{COR}=3.818,95 \%$ CI, (1.321-5.031)]; husbands who had no formal and had primary (1-8 grades) educational status being significant determinant factors for the developments of obstetric complication pregnant mothers $[\mathrm{COR}=9.321,95 \% \mathrm{CI},(2.886$, 30.11)], $[\mathrm{COR}=1.45,95 \% \mathrm{CI},(1.0424-2.53)]$; and mothers who couldn't get information regarding determinants factors of obstetric complications 16 times more likely develop obstetric complication compare to had information access, $[\mathrm{COR}=16.012$, 95\% CI (6.718-25.161)]; whereas ethnicity, religion, marital status, and monthly income in cases and controls didn't have statically significant in relation to obstetric complications ( $\mathrm{P}$ value $>0.05$ ) [Table 5]

\section{(ii) Multivariate Analysis of Distant Determinant Factors Associated with Obstetric Complication}

Variables regarding distant determinant factors which were found to be associated with the outcome variable in the bivariate analysis $(\mathrm{P} \leq 0.25)$ were taken to the multivariable analysis. This is basically to compensate for the power of the test since negative findings (that is, $p>0.05$ ) may be just 
because of inadequate power. Multivariate analysis were computed for selected variables using conditional logistic regression, variables having $\mathrm{p}$-value $\leq 0.05$ in multivariate analysis with being a case of obstetric complication on adjusted or multivariate analysis and those believed to be distant determinant factors [Table 5].

After adjusting for possible confounding factors in the matched pair conditional logistic regression regarding distant determinant factors of obstetric complications only information access concerning obstetric factors and age of first pregnancy less than 18 years had statically significant i.e. mothers who had experience of first pregnancy from less than 18 years in recent pregnancy 2.8 times more likely had obstetric complication than those mothers counter parts, $[\mathrm{AOR}=2.825,95 \% \mathrm{CI},(2.1-3.27)]$; and mothers who had no information access to determinant factors 13.7 times more likely develop obstetric complications than who had obstetric complication, $[\mathrm{AOR}=13.742,95 \% \mathrm{CI},(4.935-22.270)]$.

Table 5. Distant determinants of obstetric complications among women in Debre Berhan Referral Hospital in bivariate and multivariate analysis, Debre Berhan Town, Ethiopia, June 2019.

\begin{tabular}{|c|c|c|c|c|c|c|}
\hline Variables & Categories & $\begin{array}{l}\text { Case } n=47 \\
(\%)\end{array}$ & $\begin{array}{l}\text { Controls n=94 } \\
(\%)\end{array}$ & Crude OR (95\% CI) & Adjusted OR (95\% CI) & p-value \\
\hline \multirow{3}{*}{ Age (in years) } & $15-24$ & $10(21.3)$ & $26(27.7)$ & 1 & 1 & \\
\hline & $25-34$ & $25(53.2)$ & $58(61.7)$ & $0.56(0.054,0.973)$ & $0.72(0.543-0.856)$ & 0.75 \\
\hline & $\geq 35$ & $12(25.5)$ & $10(10.6)$ & $1.83(1.023,3.27)$ & $5.36(3.473,9.735)$ & 0.21 \\
\hline \multirow{2}{*}{$\begin{array}{l}\text { Age of first } \\
\text { marriage }\end{array}$} & $<18$ years & $11(24.6)$ & $1(1.1)$ & $0.671(0.432,0.805)$ & $2.639(1.603,11.478)$ & 0.198 \\
\hline & $\geq 18$ years & $32(74.4)$ & 87 (98.9) & 1 & 1 & \\
\hline $\begin{array}{l}\text { Age of first } \\
\text { pregnancy }\end{array}$ & $<18$ years & $9(19.1)$ & $1(1.1)$ & $8.364(8.02,9.07)$ & $2.825(2.1,27.3)$ & 0.001 \\
\hline \multirow{4}{*}{$\begin{array}{l}\text { Maternal } \\
\text { educational } \\
\text { status }\end{array}$} & No formal education & $15(32.65)$ & $10(10.5)$ & $3.818(1.321,11.031)$ & $0.168(0.012,0.286)$ & 0.319 \\
\hline & Primary (1-8 grade) & $10(19.6)$ & 17 (18.9) & $1.273(0.440,0.679)$ & $0.298(0.041,0.540)$ & 0.181 \\
\hline & Secondary (9-12 grade) & $11(29.3)$ & $39(41.1)$ & $0.718(0.273,0.882)$ & $0.221(0.044,0.427)$ & 0.229 \\
\hline & College diploma and above & $11(29.3)$ & $28(29.9)$ & 1 & 1 & 0.069 \\
\hline \multirow{3}{*}{$\begin{array}{l}\text { Husband } \\
\text { educational } \\
\text { status }\end{array}$} & No formal education & $18(42.9)$ & $7(8.0)$ & $9.321(2.886,30.11)$ & $11.839(7.885,158.293)$ & 0.313 \\
\hline & Primary (1-8 grade) & $6(14.3)$ & $15(17.0)$ & $1.45(1.0424,9.53)$ & $3.179(2.388,26.043)$ & 0.068 \\
\hline & Secondary (9-12 grade) & $10(23.8)$ & $37(42)$ & $0.98(0.343,0.997)$ & $1.875(1.392,8.965)$ & 0.281 \\
\hline \multirow{2}{*}{$\begin{array}{l}\text { Information } \\
\text { access }\end{array}$} & Yes & $13(27.7)$ & $82(87.2)$ & 1 & 1 & \\
\hline & No & $34(72.3)$ & $12(12.8)$ & $16.012(6.7,18,18.161)$ & $13.742(4.935,38.270)$ & 0.000 \\
\hline
\end{tabular}

\subsubsection{Intermediate Determinant Factors}

\section{(i) Bivariate Analysis of Intermediate Determinant Factors Associated with Obstetric Complication}

Bivariate analysis was run in the conductional logistic regression regarding intermediate determinant factors to check the association between dependent and independent variables. Accordingly variables of birth intervals $(\leq 1$ year and $\geq 4$ years), history of obstetric complication, planed pregnancy, induced labor, anemia, weight loss, FP utilization, access to ANC service, ANC utilization and access to PNC had statically significant $(\mathrm{P}<0.05)$ associated with obstetric complication, whereas rest had no statically significant [Table 6].

Accordingly, study participants who had birth interval less or equal 1 year were 2.8 times more likely to be a case of obstetric complication than those who had (2-4) years birth interval, $[\mathrm{COR}=2.83,95 \% \mathrm{CI},(1.804-3.981)]$. In the same way, mothers who had history of obstetric complications were 12 times more likely to be a case of obstetric complications than those mothers who had no history; $[\mathrm{COR}=12.00,95 \% \mathrm{CI},(2.004-23.241)]$. Regarding abortion mothers who had history abortion 6.3 times more likely have obstetric complication than those mothers who had no history; $[\mathrm{COR}=6.349,95 \% \mathrm{CI},(2.642-11.823)]$.

Mothers who had no planned pregnancy were 3.23 times more likely to be a case of obstetric complication than those who had no, $[\mathrm{COR}=3.23,95 \% \mathrm{CI}$, (1.519- 5.870)]. Similarly mothers who had history of anemia and weight loss in the current pregnancy were 6.5 times and 15.5 times more likely develop obstetric complications than those who had no history, $[\mathrm{COR}=6.5,95 \% \mathrm{CI},(3.005-10.035)],[\mathrm{COR}=15.5$, $95 \% \mathrm{CI},(10.039,20.615)]$ respectively. With regard to FP utilization and access to ANC service mothers who didn't use FP methods had 9.5 more likely develop obstetric complications, $[\mathrm{COR}=9.5,95 \% \mathrm{CI},(3.449-12.362)]$ than who used. Similarly mothers who had no access to ANC service had 45.5 times develop obstetric complications compare to who didn't have, [COR=45.484, 95\% CI, (5.77193.58)].

There was $2.2 \%$ reduced risk of developing obstetric complication among mothers who used focused ANC service based compared to those who didn't use, [COR=0.022 95\% CI, (0.005-0.090)]. Similarly mothers who used PNC services were $24 \%$ reduced risk of developing obstetric complications than who didn't $[\mathrm{COR}=0.24,95 \% \mathrm{CI},(0.107$ $0.538)]$.

\section{(ii) Multivariate Analysis of Intermediate Determinant Factors Associated with Obstetric Complication}

After adjusting for possible confounding factors in the matched pair conditional logistic regression regarding intermediate determinant factors birth intervals 1 year and less, mother who had history of previous obstetric complications, induced pregnancy, anemia and focused ANC utilization had significant association with obstetric complications [Table 6]. 
Accordingly mothers who had birth interval 1 year and less were 2.58 times more likely to be a case of obstetric complication than those who had 2-4 years birth interval, $[\mathrm{AOR}=2.581,95 \% \mathrm{CI},(1.679-3.896)]$; and mothers who had history of obstetric complications 3.5 times more likely to be a case of obstetric complications than those mothers who had no history, $[\mathrm{AOR}=3.450,95 \% \mathrm{CI},(2.001-4.155)]$. Mothers with a history of anemia had 3.1 higher odds of having obstetric complications compared to mothers with no history of previous anemia [AOR=3.1, 95\% CI, (2.002-4.414)]. Mothers who used focused ANC prevent obstetric complications by $30 \%$ as compare to who hadn't used focus ANC $[\mathrm{AOR}=0.30,95 \% \mathrm{CI},(0.014-0.877)]$. The odds of having obstetric complication among mothers who had history of induced labor were 4.9 times that of those who had not induced labor $[\mathrm{AOR}=4.9,95 \%$ CI 2.008-6.321)].

Table 6. Intermediate determinants of obstetric complication among women in Debre Berhan Referral Hospital in bivariate and multivariate analysis, Debre Berhan Town, Ethiopia, June 2019.

\begin{tabular}{|c|c|c|c|c|c|c|}
\hline Obstetric factors & Categories & Case $n=47(\%)$ & Controls n=94 (\%) & Crude OR (95\% CI) & Adjusted OR (95\% CI) & p-value \\
\hline \multirow{3}{*}{ Birth interval } & $\leq 1$ year & $10(21.7)$ & $4(4.2)$ & $2.833(1.804,3.981)$ & $2.581(1.673,3.896)$ & 0.000 \\
\hline & 2-4 year & $7(14.8)$ & $56(60)$ & $0.119(0.045,0.311)$ & $0.076(0.021,0.273)$ & 0.167 \\
\hline & $\geq 4$ years & $30(63.8)$ & $34(35.2)$ & & & 0.000 \\
\hline \multirow{2}{*}{$\begin{array}{l}\text { History of previous } \\
\text { obstetrics complication }\end{array}$} & Yes & $13(26.7)$ & - & $12.00(2.004-23.241)$ & $3.450(0.001,0.155)$ & .0 .000 \\
\hline & No & $34(73.9)$ & $94(100)$ & 1 & 1 & \\
\hline \multirow{2}{*}{ History of abortion } & Yes & $7(13.0)$ & $5(16.3)$ & $6.349(2.642-11.823)$ & $1.652(0.099,0.497)$ & 0.726 \\
\hline & No & $40(87)$ & $29(93.7)$ & 1 & 1 & \\
\hline History of still birth & No & $43(93.5)$ & $91(98.9)$ & 1 & 1 & \\
\hline \multirow{2}{*}{ Planned pregnancy } & Yes & $25(52.2)$ & $73(77.9)$ & 1 & 1 & \\
\hline & No & $22(47.8)$ & $21(22.1)$ & $3.23(1.519,6.870)$ & $0.501(0.093,2.693)$ & 0.421 \\
\hline \multirow{2}{*}{ Pregnancy induced } & Yes & $12(26.5)$ & $2(2.1)$ & $6.1(0.013,0.286)$ & $4.90(0.008,0.321)$ & 0.002 \\
\hline & No & $35(73.9)$ & $92(97.9)$ & 1 & 1 & \\
\hline \multirow{2}{*}{ Anemia } & Yes & $10(19.6)$ & - & $6.5(3.005,10.035)$ & $3.1(1.002,5.414)$ & 0.009 \\
\hline & No & $37(80.4)$ & $94(100)$ & 1 & 1 & \\
\hline Weight loss & No & $38(82.6)$ & $92(96.8)$ & 1 & 1 & \\
\hline \multirow{2}{*}{ FP utilization } & Yes & $29(60.9)$ & $88(93.7)$ & 1 & 1 & \\
\hline & No & $18(39.1)$ & $6(6.3)$ & $9.5(3.449,26.362)$ & $6.77(2.919,9.864)$ & 0.060 \\
\hline \multirow{2}{*}{ Access to ANC service } & Yes & $32(67.4)$ & $93(98.9)$ & 1 & 1 & \\
\hline & No & $15(32.6)$ & $1(1.1)$ & $45.484(5.771,93.58)$ & $7.73(4.32,7.56)$ & 0.09 \\
\hline \multirow{2}{*}{ Focused ANC utilization } & Yes & $23(49)$ & $93(98.9)$ & $0.022(0.005,0.098)$ & $0.30(0.014,0.877)$ & 0.014 \\
\hline & No & $24(51)$ & $1(1.1)$ & 1 & 1 & \\
\hline \multirow{2}{*}{ Access to PNC } & Yes & $34(71.7)$ & $93(98.9)$ & 1 & 1 & \\
\hline & No & $13(28.3)$ & $1(1.1)$ & $37.03(4.662,294.12)$ & $1.16(1.020,5.799)$ & 0.942 \\
\hline \multirow{2}{*}{ PNC utilization } & Yes & $10(21.7)$ & $51(53.7)$ & $0.24(0.107,0.538)$ & $0.616(0.159,0.987)$ & 0.422 \\
\hline & No & $37(78.3)$ & $43(46.3)$ & 1 & 1 & \\
\hline
\end{tabular}

\section{Discussion}

This study was conducted to identify determinant factors associated with obstetric complications among women in Debre Berhan Referral Hospital, North Shewa, Ethiopia. This particular study identifies distant and intermediate determinants of obstetric complications among pregnant and postpartum women.

In current study income and marital status of mothers didn't have statically significant (P-value $>0.05$ ) associated with obstetric complications but the study conducted in Geneva in 2015 on immigrants showed that women facing in low income community were at 17 fold higher risk for obstetric complications. In the same study women without marital support were at 7 fold higher risk for obstetric complications [21]. Similarly the study conducted in India 2015 shows that women with sever obstetric complications came from families classified as living low income which was significant. This discrepancy might be due to that now a day the obstetric service in Ethiopia are free so no need of income access and in this study the participants were dwellers so they might need less marital support than immigrants [7].

The current study result showed that age of pregnant mothers 35 and above were had significant association with obstetric complications. The odds of Pregnant women with age 35 and above to have obstetric complications were 1.8 times $[\mathrm{COR}=1.83,95 \% \mathrm{CI},(1.023-3.27)]$ higher compared to their counter parts of women less than 35 years. This is in line with a previous finding a case-control study findings conducted in Debre Tabor town, Northwest Ethiopia in 2017 on factors associated with obstetric complication which shows that advanced maternal age was found to be significantly associated with higher odds of obstetric complications [20]. This in line might be due to the physiological aging process in the reproductive organs.

Regarding this study mothers who had birth interval less and equal 1 year were 2.83 times more likely to be a case of obstetric complication than those who had 2-4 years birth interval, $[\mathrm{COR}=2.83,95 \% \mathrm{CI},(1.804-3.981)]$. This current findings is consistent with the previous findings in traditional unmatched design in USA 2017 which showed that short inter pregnancy intervals were significantly associated with 
increased risk of obstetric complications [22]. This consistence might be due to that shorter birth interval leads to lack of preparedness and maturity of reproductive organs.

In current study mothers who had experience of first pregnancy from less than 18 years in current pregnancy more likely had 2.8 times obstetric complication than those mothers who had first pregnancy experience 18 and above years, $[\mathrm{AOR}=2.825,95 \% \mathrm{CI},(2.1-3.27)]$. This findings is in line with the findings in India a hospital based cross-sectional study on Socio-demographic determinants of obstetric complications 2016 which showed that the odds of age at first pregnancy less than 18 years was 2.35 times more likely to develop obstetric complications. This finding might be due to inadequate growth of reproductive organ especially in the early reproductive age [23].

In this study mothers who had history of obstetric complications 3.5 times more likely to be a case of obstetric complications than those mothers who had no history, $[\mathrm{AOR}=3.450,95 \% \mathrm{CI},(2.001-4.155)]$. This finding is consistent with finding in Jimma University Specialized Hospital in 2016 which show that, women who had history of obstetric complications 18 times more develop obstetric complications [24]. This finding also consistent with study conducted in in maternity centre of District Hospital Ujjain, Madhya 2015 which shows that history of previous obstetric complications was 2.28 times more risky of having current obstetric complications [25]. This consistency also might be due to that mothers who had previous obstetric complications might vulnerable to physiological and pathological change of reproductive systems.

Regarding abortion mothers who had history abortion 6.3 times more likely had obstetric complication than those mothers who had no history, [COR $=6.349,95 \%$ CI, (2.64211.823)]. This findings is consistent with the findings of study conducted in Addis Ababa Ethiopia 2017 which show that history of abortion had statically significant associated with obstetric complications [26]. This might be due to hormonal and pathological change of reproductive systems.

The current study showed that mothers who had no formal education 3.8 more likely develop obstetric complications than mothers who had formal education, COR and 95\% CI, 3.818 (1.321-5.031). This finding is consistent with the previous study findings in a case control study findings conducted in Debre Tabor town, Northwest Ethiopia 2017 on factors associated with obstetric complication which shows that mothers with no formal education were 2 times more likely to develop an obstetric complications. This might be due to mother had not appropriate knowledge on danger signs during pregnancy, if mother had not appropriate knowledge on danger signs during pregnancy, they didn't want to seek health care and couldn't easily identify those problems. But in this study primary education had no statically significant whereas in Debre Tabor town mothers with primary education were 1.6 times more likely to have an obstetric complication compared to those in higher education [20]. This inconsistence might be due to getting more health education in current study.
This study also show that mothers who couldn't get information regarding determinants factors of obstetric complications 16 times more likely develop obstetric complication compare to had information access, $[\mathrm{COR}=16.012,95 \% \mathrm{CI}(6.718-25.161)]$. In adjusted analysis mothers who had no information access regarding obstetrics also 13.7 times more likely develop obstetric complications than who had obstetric complication, $[\mathrm{AOR}=13.742,95 \% \mathrm{CI}$, 4.935-22.270)]. This is consistent with the findings in in study conducted in Gamo Gofa Zone, Southern Ethiopia which show that pregnant women who didn't have information access regarding danger signs during pregnancy 102 times more likely to have obstetric complications [19]. This indicates that getting information about pregnancy and its consequences for mother has its own effect on their reduction on developing obstetric complications.

Regarding planned pregnancy this study shows that mothers who had no planned pregnancy were 3.23 times more likely to be a case of obstetric complication than those who had no, $[\mathrm{COR}=3.23 \quad 95 \%$ CI, $(1.519-6.870)]$. This finding is in line with the findings in facility based case control study, on determinants of obstetric complications among Mothers who Gave Birth at Hospitals in Gamo Gofa Zone, Southern Ethiopia at 2017 which showed that the odds of mothers who didn't planned the current pregnancy were 8.5 more likely develop obstetric complications [19]. This might be due to that mothers keep adequate birth intervals and obstetric care decrease hormonal, pathological and immaturity of reproductive system.

Finding of the present study is that mothers who had history of anemia and weight loss in the current pregnancy were 6.5 times and 15.5 times more likely develop obstetric complications than those who had no history, $[\mathrm{COR}=6.5$, 95\% CI, (3.005, 10.035)], [COR=15.5, 95\% CI, (10.039, $20.615)]$ respectively. This is consistent with the findings in a nested case-control study on distant and proximate factors associated with maternal sever obstetric complication in selected public hospitals of Addis Ababa, Ethiopia in 2017 which shows that mothers who had history of anemia and weight loss had significant association with obstetric complications [26]. Similarly it is consistence with study conducted in Jimma University Specialized Hospital in 2016 [24]. This consistency might be due to that mothers with anaemic and low weight may decrease blood flow to reproductive organs, so if there reduction of blood flow the reproductive organs might be immature pathologically to survive pregnancy process.

With regard to access to and utilization of ANC service mothers who had no access to ANC service had 45.5 times develop obstetric complications compare to who didn't have, $\mathrm{COR}=45.484,95 \% \mathrm{CI},(5.771-13.58)]$. There was also $2.2 \%$ and $30 \%$ reduced risk of developing obstetric complication among mothers who used focused ANC service compared to those who didn't use, $(\mathrm{COR}=0.022,95 \% \mathrm{CI},(0.005-0.098)]$, $(\mathrm{AOR}=0.30,95 \% \mathrm{CI}, 0.014-0.877)]$ in crude and adjusted analysis respectively. This is consistent with the findings in an observational follow-up study conducted from 1st April to 
30th June 2015 in maternity centre of District Hospital Ujjain, Madhya Pradesh which shows that complications of pregnancy was found to be significantly associated with those who had no history of any antenatal care visit, these participants showed the 2.28 times risk of having obstetric complications [25]. Similarly a case-control study on factors associated with obstetric complications at UK in 2015 shows that the odds ratio of inadequate use of antenatal care was 15 times higher among women who had sever obstetric complications a compared with women who survived [27]. This finding also consistent with study conducted in Addis Ababa [26], and in Jimma University Specialized Hospital in 2016 mothers who attend ante natal care (ANC) were 83\% less likely to obstetric complication [24]. This might be due to that ANC attendance makes the women to prepare for birth and for complication readiness.

With regard to FP utilization mothers who didn't use FP methods had 9.5 more likely develop obstetric complications, $\mathrm{COR}=9.5,95 \% \mathrm{CI},(3.449-26.362)]$. This finding is in line with in facility based case control study, on determinants of obstetric complications among Mothers who Gave Birth at Hospitals in Gamo Gofa Zone, Southern Ethiopia at 2017 which showed that mothers who didn't use FP methods were 9 times more likely develop obstetric complications [19]. This finding indicated that if mothers didn't have pregnancy, they couldn't face to developing obstetric complications.

This study has its own strengths and limitations. The fact that study used primary data that is factors were collected directly from mothers by interviews and matching by residence and parity was employed among the cases and controls enabled to maintain its validity and minimize confounding factors. The limitation could be recall and social desirability biases (might mask the association between the risk factors identified and being a case of obstetric complications) among the study participants when reporting some behavioral risk factors.

\section{Conclusions and Recommendations}

\subsection{Conclusion}

Hemorrhage, Obstructed labor, Pregnancy induced hypertension, Hyper emesis gravid arum, Uterine rapture, and Puerperal sepsis were major Obstetric complications in Debre Berhan Referral Hospitals. Mothers with obstetric complications had more history of previous obstetric complications, history of abortion, history of still births, history anemia and weight loss than those without obstetric complications. Mothers who had no formal education, whose husband educational status were had no formal education and primary (1-8 grades), with history of first pregnancy less than 18 years in recent pregnancy, and who didn't get information access regarding obstetric complications were distant determinant factors and more risky to develop obstetric complications. Birth interval less and equal 1year, history obstetric complications, planed pregnancy, anemia, weight loss, access to FP service, access to ANC service and access to PNC service were intermediate determinants to develop obstetric complications.

\subsection{Recommendations}

All effort must be put in place to reduce those distant and intermediate determinant factors for developments of obstetric complications.

1. At national level the government should revise and doing more strategies, policies and activates for mothers to get enough information, services (ANC, PNC and FP) and treatments in their nearby health facilities as well as special co-work with NGOs and other government sectors. In the same way at national level detail operational research should be performed in each obstetric case on their determinant factors and appropriate intervention should be applied.

2. In the regional (Amhara) level the regional health bureau should apply and follow how the strategies of minimizing obstetric complications performed in each zonal administrates. In the same way it should work with educational health bureau to empower women and their husbands, give consecutive trainings to health professionals on the way of preventing the determinants of obstetric complications.

3. At zonal (North Shewa) and near woredas levels the administrative staffs should give consecutive training and follow up on focused ANC, FP methods and PNC services to health professionals; and full fill health education guides on how to prevent and minimize determinants of obstetric complications.

4. At Debre Berhan referral hospital and near Health facilities level should give consecutive health education to women; give focused ANC and regular PNC follow up; promote and facilitate utilizations of FP; and follow and supervise health professionals on the way of how to prevent and minimize obstetric complications

5. Health professionals should treat and follow up chronic health conditions early to not to be the risk; follow and aware mothers with previous obstetric complications, had history of abortions and history of still birth, promote and facilitate utilizations of modern contraceptive methods; and provide structured based health education during the ANC period, knowledge of events and danger signs during pregnancy time and labor. They also should follow, prevent and treat anemia.

6 . Women in the reproductive age group should plan their pregnancy, keep birth interval 2 up to 4 years, use focused ANC, use modern contraceptive methods, use PNC follow up, and minimize Anaemia and weight loss by taking nutritional support like Iron and additional meals.

\section{Lists of Abbreviations}

AFI:-Acute Febrile illness

ANC:-Antenatal Care

APH:-Antepartum Haemorrhage

BEmOC:-Basic Emergency Obstetric Care 


\author{
CEmOC:- Comprehensive Emergency Obstetric Care \\ CI:- Confidence Interval \\ CPD:- Cephalo-Pelvic Disproportion \\ DBRH:- Debre Berhan Referral Hospital \\ EDHS:- Ethiopia Demographic and Health Survey \\ ETBR:- Ethiopian Birr \\ FP:- Family Planning \\ Hct:-Haematocrit \\ MCH:-Maternal and Child Health \\ MDGs:-Millennium Development Goals \\ MDSR:-Maternal Death Surveillance and Response \\ MMR:-Maternal Mortality Ratio \\ MOH:-Ministry Of Health \\ NGOs:-Non-Governmental Organizations \\ PNC:-Postnatal Care \\ PPH:-Postpartum Haemorrhages \\ PROM:-Premature Rupture of Membrane \\ SVD:-Spontaneous vaginal delivery \\ WHO:-World Health Organization
}

\section{Declarations}

\section{Ethics Approval and Consent to Participate}

Prior to starting the study, the final proposal was submitted to Department of public health, College of Health Science, Debre Berhan University for ethical clearance and approval. Then letter of permission was obtained from the Ethical review committee of Department of public health, College of Health Science, Debre Berhan University. After explaining about the purpose of the study, and the possible benefit of the study; oral permission was obtained from the hospital director before proceeding. Respect and full confidentiality of the study participant's response was granted since only the investigator was having access to the data. Then information was collected after securing oral consent from study participant. Individual Verbal consent was secured as well.

\section{Consent for Publication}

'Not applicable'

\section{Availability of Data and Materials}

The finding of this study is generated from the data collected and analyzed based on stated methods and materials. The original data supporting this finding are available from the corresponding author on reasonable request.

\section{Competing Interests}

"The authors declare that they have no competing interests"

\section{Funding}

'Not applicable'

\section{Authors' Contributions}

$\mathrm{AB}$ participated in the design of the study, performed the data collection and the statistical analysis and served as the corresponding author of the manuscript. RW and ZK supervised the study, ensured quality of the data, assisted in the analysis and interpretation of the data. All authors read and approved the manuscript.

\section{Authors' Information}

$\mathrm{AB}$ is Primary Healthcare unit Director at Kotu, North Shewa Zone, Amhara Regional State, Ethiopia

RW is Maternal and Child Health (MCH) Program Coordinator, SendafaBeke Town, Ethiopia.

ZK is Water, Sanitation, Hygiene (WASH) and Neglected Tropical Diseases (NTDs) Program Coordinator, Nekemte town, Western Ethiopia.

\section{Acknowledgements}

We are grateful to the managers and the staffs of Debre Berhan Referral Hospital for their cooperation and allowing us to conduct my research in the hospital.

\section{References}

[1] CDC. Division of Reproductive Health, National Center for Chronic Disease Prevention and Health Promotion, 2018.

[2] CDC. Severe Maternal Morbidity in the United States. Archived from the original on 2015.

[3] CDC. The Society of Obstetricians and Gynecologists of Canada (SOGC). Severe Maternal Morbidity. Canada, 2016.

[4] Gabrysch S. The determinants of delivery service use, BMC Pregnancy Childbirth, 9, 34 (2015).

[5] Kassebaum N, Bertozzi-Villa A, Coggeshall MS, et al. Global, regional, and national levels and causes of maternal mortality, 2015.

[6] Ramatu A, Yohanna S. Pattern and determinants of obstetric complications among women delivered at Yusuf Dan-Tsoho Memorial Hospital. Tudunwada, India, 2014.

[7] Gogoi M. Pregnancy Complications and Birth Outcome: Do Health Care Services Make a Difference? Mumbai, Uganda, 2015.

[8] Annettee N, Sarah N, Othman K, Michael O, Scovia N, Rose C, Nelson K, Dan K. Maternal near misses from two referral hospitals in Uganda: a prospective cohort study on incidence, determinants and prognostic factors. Uganda, 2016.

[9] Federal Ministry of Health Maternal Death Surveillance and Response (MDSR) Technical Guideline. Addis Ababa, Ethiopia, 2014.

[10] Ethiopian public health institute. National Maternal Death Survellance and Response annual report, 2016.

[11] World Bank. Safe motherhood and maternal health. http://go.worldbank.org/V5EPGZUL4. Accessed October 20, 2013.

[12] Muh I, Yadi Y. Risk factors associated with maternal mortality determinants. Gowa, Indonsia, 2015. 
[13] Vanden N, Graham WJ. Quality of care for maternal and new born health: India, 2014.

[14] World Health Organization (WHO), Beyond the numbers: Reviewing maternal deaths and complications to make pregnancy safer, 2014.

[15] Katherine E. Severe Complications for Women during Childbirth, USA, 2017.

[16] United Nations Population Fund. Maternal health, 2017.

[17] GBD. Global, regional, and national age-sex specific all-cause and cause-specific mortality, 2013.

[18] Debre Berhan Referral Hospital first quarter report, Debre Berhan Town, Ethiopia, 2019.

[19] Feleke G. Teklemariam G. Gemechu K. Desta H. Yinager W. Determinants of Obstetric complications among Mothers who Gave Birth at Hospitals in Gamo Gofa Zone, Southern Ethiopia. (2017) 25 (5): 259-266).

[20] Abraham S. Achenef A. Amelework G. Factors associated with adverse pregnancy outcome. Debre Tabor town, Northwest Ethiopia, 2018.

[21] Emily W, Pauline M, Katherine L. "The Effect of maternal chronic disease on obstetric complications in twin pregnancies. USA." 2014.
[22] Lindsay K, Tyler A, Michele H, Vanessa K. "Obstetric Outcomes and Delivery-Related Health Care Utilization and Costs among Pregnant Women with Multiple Chronic Conditions. USA, 2018.

[23] Reena R, Umadevi N, Ajitha B. SEVERE OBSTETRIC MORBIDITY: PREVALENCE, RISK FACTORS AND OUTCOME. National Journal of Community Medicine, India, Apr-Jun 2015.

[24] EyosiyasY, Nigus A, Mulumebet A, Amanuel T. "Determinants of Adverse Pregnancy Outcomes among mothers. Jimma University Specialized Hospital, Ethiopia." 2016.

[25] Suchita S, Kirti D, Dharmpal S, Deepika B. "A Hospital Based Study on Complications of Childbirth and Associated Risk Factors at Ujjain, Madhya Pradesh." 2015.

[26] Ewnetu F, Alemayehu W, Mesganaw F, Birgitta E. "Distant and proximate factors associated with maternal near-miss: a nested case control Study in selected public hospital. Addis Ababa, Ethiopia." 2017.

[27] Nair M, Kurinczuk JJ, Brocklehurst P, Sellers S, Lewis G, Knighta M. "Factors associated with maternal death from direct pregnancy complications: a UK national case-control study." 2015. 